\title{
Blood Group Antigens on Fetal Red Cells Obtained by Umbilical Vein Puncture under Ultrasound Guidance: A Rapid Hemagglutination Test To Check for Contamination with Maternal Blood
}

\author{
BAHMAN HABIBI, MARYSE BRETAGNE, YVES BRETAGNE, FRANÇOIS FORESTIER, AND \\ FERNAND DAFFOS \\ National Blood Transfusion Center, Service de Biologie and Department of Prenatal Diagnosis and Fetology, \\ Hôpital Notre Dame de Bon Secours, Paris, France
}

\begin{abstract}
Seventy-two fetal blood samples obtained by umbilical vein puncture under ultrasound guidance for prenatal diagnosis and monitoring purposes were tested for the expression of 38 blood group antigens. The gestational age at sampling ranged from 18 to 34 wk with $76 \%$ of fetuses between 20 and 25 wk. Compared to adult red blood cells, the following antigens showed either abnormally decreased frequency, or significantly reduced expression: $A, B, A_{1}, H, L^{a}, L^{b}, L^{a}, L^{b}, P_{1}, P$, and I. Selected anti$I$ and anti-i cold agglutinins, active at room temperature, were used at appropriate dilution, in a rapid slide or spin test to check for possible mixture of the sample with maternal blood and were shown to detect contaminations up to $5 \%$. The test has proved particularly helpful for immediate and in process assessment of foetal origin of the sampled blood. (Pediatr Res 20:1082-1084, 1986)
\end{abstract}

\section{Abbreviations}

BG, blood group

RBC, red blood cells

Fetal BG studies have so far been performed on cord samples taken in cases of therapeutic abortion either in occasional circumstances (1) or in series such as those reported by Cleghorn (unpublished observations cited in Reference 1), Toivanen and Hirvonen (2), and Mackenzie et al. (3). These studies have already highlighted the fact that the antigenic make-up of the adult RBC membrane is not achieved at the same developmental stage. Some antigens such as rhesus which is determined by protein structures are well developed as early as the 6th gestational wk, while others such as A, B, H, Lewis, I, which are determined by immunodominant sugars and require simple or multiple transferase enzymes for their development, are not fully expressed at birth.

The current availability of numerous fetal blood samples collected in utero in pregnant women for diagnosis and monitoring purposes led us to design an extensive study of $B G$ antigens of these fetuses on a systematic basis under standardized conditions in order to supplement the already available data on the development of BG markers in man. As a byproduct of this investigation we studied whether the appropriate use of antisera to

Received October 28, 1985; accepted June 4, 1986

Correspondence and reprint requests Dr. Bahman Habibi, National Blood Transfusion Center, 53 boulevard Diderot, 75012 Paris, France. antigens I and $i$ which are among the specific markers of respectively adult and fetal RBC could prove of any appreciable help to check for accidental contamination of the fetal sample by blood aspirated from maternal vessels.

\section{MATERIALS AND METHODS}

Fetal blood was obtained in 72 pregnant women for prenatal diagnosis and monitoring purposes. Two $\mathrm{ml}$ of blood were aspirated from the umbilical vein at the placental cord insertion using a 20 -gauge needle guided by ultrasound as described previously (4). The gestational age at sampling is shown in Figure 1 where 55 fetuses $(76.6 \%)$ were 20 to $25 \mathrm{wk}, 4(5.5 \%) 18$ to 20 wk, and 13 (18\%) 26 to 34 wk.

$\mathrm{RBC}$ were frozen in liquid nitrogen immediately after sampling. The samples could thus be thawed simultaneously and tested with the same reagents under homogenous and standardized conditions.

Seventy-two cord blood samples taken at delivery from fullterm babies were processed in a similar way but were tested only for antigens showing immature development on fetal RBC (Fig. 2).

RBC phenotyping was performed using 38 antisera of different specificities and appropriate positive and negative adult RBC controls by conventional hemagglutination techniques (saline, indirect Coombs' on protease treated or untreated RBC according to antisera). Antigenic expression was graded according to the strength of agglutination obtained by undiluted antiserum and by $1: 2$ titrating dilutions thereof. The following agglutination scores were used throughout the study: 10: one or several large agglutinates, no or few free cells; 8: large agglutinates in a sea of small clumps and some free cells; 5: small agglutinates of approximately 20 cells visible macroscopically; 2 : small agglutinates visible only microscopically; 0 : no visible agglutination.

Titration score of each RBC sample was developed by summing the scores obtained at each dilution of the reagent. Scores below $70 \%$ of the adult controls run in parallel were considered to reflect weak reactivity and immature development of the antigen.

Since antigens I and $\mathrm{i}$ are easily accessible and clear-cut markers of adult and fetal RBC, following preliminary tests two sera with potent anti-I and anti-i antibodies from patients with chronic cold agglutinin disease were selected. These were appropriately diluted with serum from group $A B$ donors in order to get rid of anti-A and $-B$ antibodies simultaneously present and to obtain clear-cut positive and negative reactions at room temperature with adult and cord $\mathrm{RBC}$. The reagents were then divided into $0.5-\mathrm{ml}$ aliquots and kept frozen at $-30^{\circ} \mathrm{C}$ until use. 
Adult and cord $\mathrm{RBC}$ were mixed at increasing proportions as shown in Table 2. A $5 \% \mathrm{v} / \mathrm{v}$ suspension in saline of these mixtures was added to an equal volume of the antiserum at room temperature either on a slide or in a test tube and centrifuged at $1000 \mathrm{cpm}$ for $30 \mathrm{~s}$. Reactions were read macroscopically

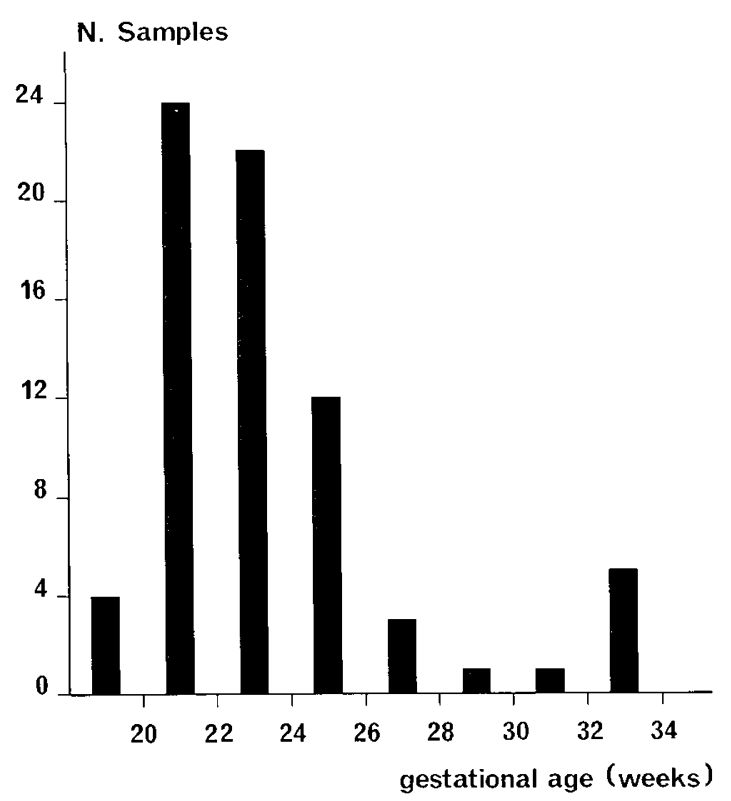

Fig. 1. Gestational age of fetuses at blood sampling. and microscopically and inspected for the presence of mixed field pattern of agglutination.

\section{RESULTS}

The panel of reagents used for blood grouping included antisera to the following $38 \mathrm{BG}$ antigens. Polymorphic antigens: A, $\mathrm{A}_{1}, \mathrm{~B}, \mathrm{D}, \mathrm{C}, \mathrm{C}^{\mathrm{w}}, \mathrm{c}, \mathrm{E}, \mathrm{e}, \mathrm{K}, \mathrm{k}, \mathrm{Kp}^{\mathrm{a}}, \mathrm{Fy}^{\mathrm{a}}, \mathrm{Fy}^{\mathrm{b}}, \mathrm{Jk}^{\mathrm{a}}, \mathrm{Jk}^{\mathrm{b}}, \mathrm{M}, \mathrm{N}, \mathrm{S}, \mathrm{s}$, $\mathrm{Lu}^{\mathrm{a}}, \mathrm{Lu}^{\mathrm{b}}, \mathrm{Le}^{\mathrm{a}}, \mathrm{Le}^{\mathrm{b}}, \mathrm{P}_{1}, \mathrm{Xg}^{\mathrm{a}}$. Monomorphic antigens: $\mathrm{H}, \mathrm{Rh} 17$, $\mathrm{Kp}^{\mathrm{b}}, \mathrm{Js}^{\mathrm{b}}, \mathrm{Fy}^{3}, \mathrm{Jk}^{3}, \mathrm{P}, \mathrm{I}, \mathrm{i}, \mathrm{Ve}^{\mathrm{a}}, \mathrm{Ge}^{\mathrm{a}}$, Emma.

The strength of reaction and frequency of these antigens were identical to adult controls except for the following: $A, A_{1}, B, H$, $\mathrm{Le}^{\mathrm{a}}, \mathrm{Le}^{\mathrm{b}}, \mathrm{Lu}^{\mathrm{a}}, \mathrm{Lu}^{\mathrm{b}}, \mathrm{P}_{1}, \mathrm{P}, \mathrm{I}, \mathrm{i}$. These antigens were tested for among the 72 cord blood samples from full-term babies. The results are shown in Figure 2. It may be seen that the above antigens are either unexpressed, underrepresented, or weakly developed in fetal RBC. The findings in cord blood samples indicate, as expected, an intermediate developmental stage between fetal and adult conditions. The expression of antigen $\mathrm{H}$ is known to vary according to the ABO group in adults. Table 1 provides detailed evidence on the immaturity of this antigen in fetal RBC.

Using the selected anti-I and anti-i reagents described in "Materials and methods" against various proportions of adult and cord blood, as indicated in Table 2, a mixed field pattern of agglutination was observed even in $95 \% / 5 \%$ mixtures. Contaminations as small as $5 \%$ could therefore be identified by simple inspection.

\section{DISCUSSION}

The present study conducted in the same laboratory under homogenous and standardized conditions confirms the following

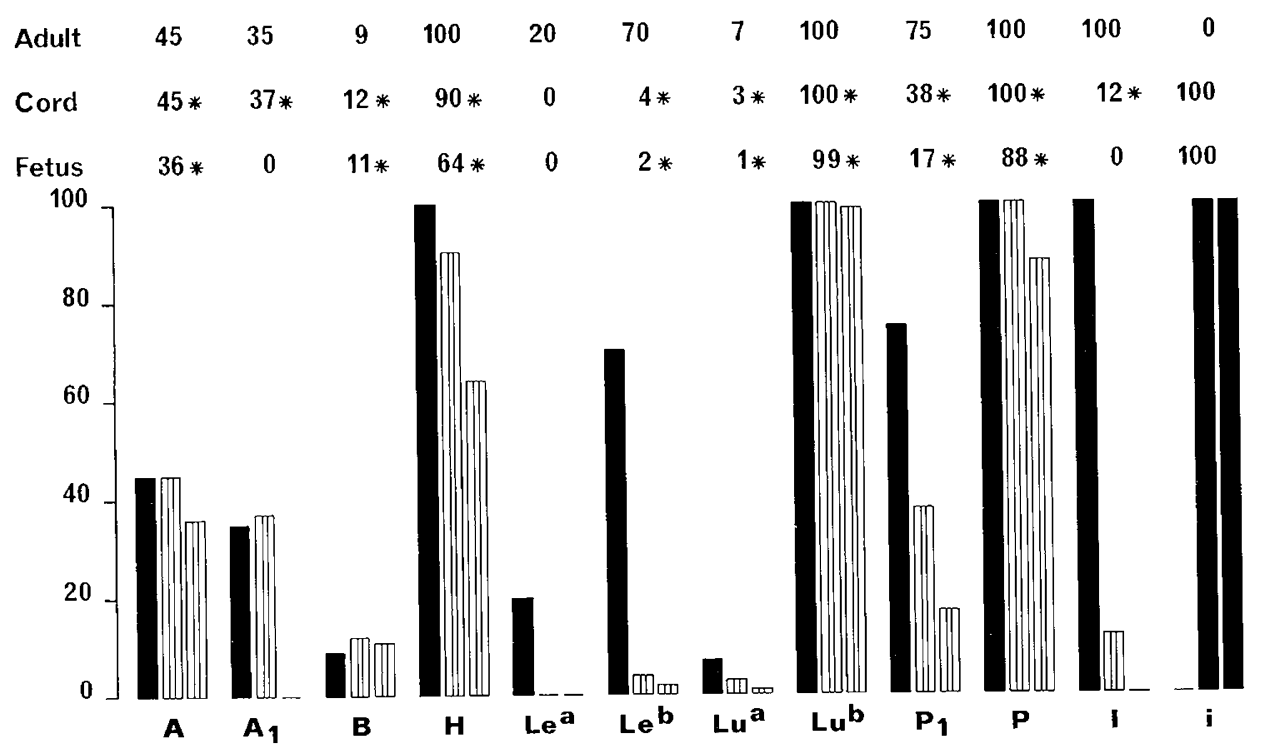

Fig. 2. Frequency and reactivity of blood group antigens identified as showing immaturity at birth. Asterisk and hatched bars indicate that a variable proportion of these samples exhibited weak reactivity. Black bars indicate normal antigenic strength. Figures indicate the percentage frequency of antigens and bars represent from left to right adult, cord, and fetal bloods.

Table 1. Percentage values indicating reduced expression of $H$ antigen in fetal samples compared to adults according to ABO blood groups

\begin{tabular}{|c|c|c|c|c|c|c|}
\hline \multirow[b]{3}{*}{ Blood group } & \multicolumn{3}{|c|}{ Fetal samples } & \multicolumn{3}{|c|}{ Adult controls } \\
\hline & \multicolumn{2}{|c|}{ Positive } & \multirow[b]{2}{*}{ Negative } & \multicolumn{2}{|c|}{ Positive } & \multirow[b]{2}{*}{ Negative } \\
\hline & Strong & Weak & & Strong & Weak & \\
\hline $\mathrm{O}$ & 80 & 20 & 0 & 100 & 0 & 0 \\
\hline A & 4 & 28 & 68 & 20 & 80 & 0 \\
\hline$B$ & 0 & 16 & 84 & 0 & 100 & 0 \\
\hline
\end{tabular}


Table 2. Agglutination of mixtures of adult and cord RBC obtained by the selected anti-I and anti-i reagents*

\begin{tabular}{|c|c|c|c|c|c|c|c|c|c|c|c|c|c|}
\hline \multicolumn{14}{|c|}{ RBC mixture } \\
\hline$\%$ adult & 100 & 95 & 90 & 80 & 70 & 60 & 50 & 40 & 30 & 20 & 10 & 5 & 0 \\
\hline$\%$ cord & 0 & 5 & 10 & 20 & 30 & 40 & 50 & 60 & 70 & 80 & 90 & 95 & 100 \\
\hline \multicolumn{14}{|c|}{ Reaction pattern } \\
\hline Anti-I & $\mathrm{P}$ & $\mathrm{P}$ & $\mathrm{P}$ & MF & MF & MF & MF & MF & MF & MF & MF & MF & $\mathrm{N}$ \\
\hline Anti-i & $\mathrm{N}$ & MF & MF & MF & MF & MF & MF & MF & MF & MF & $\mathbf{P}$ & $\mathrm{P}$ & $\mathbf{P}$ \\
\hline
\end{tabular}

* $\mathrm{P}$, strongly positive; $\mathrm{MF}$, mixed field pattern; $\mathrm{N}$, negative.

observations made in occasional circumstances (1) or in limited series $(2,3)$ by previous authors.

The antigens D, C, c, E, e, K, k Kp ${ }^{\mathrm{a}}, \mathrm{Kp}^{\mathrm{b}}, \mathrm{Fy}^{\mathrm{a}}, \mathrm{Fy}^{\mathrm{b}}, \mathrm{Jk}^{\mathrm{a}}, \mathrm{Jk}^{\mathrm{b}}$, $\mathrm{M}, \mathrm{N}, \mathrm{S}, \mathrm{s}, \mathrm{Xg}^{\mathrm{a}}, \mathrm{Ve}^{\mathrm{a}}$, and $\mathrm{Ge}^{\mathrm{a}}$ are fully developed in the second half of pregnancy since their strength and frequency are similar to those found in adults. The presence of IgG antibodies to these antigens in mother triggered by previous pregnancies or blood transfusions may cause hemolytic disease of fetus in the second half of intrauterine life if not earlier.

Antigens I, $\mathrm{Le}^{\mathrm{a}}, \mathrm{Le}^{\mathrm{b}}$ are not expressed on fetal $\mathrm{RBC}$ and antigens $\mathrm{A}, \mathrm{B}, \mathrm{H}, \mathrm{P}_{1}, \mathrm{P}, \mathrm{Lu}^{\mathrm{a}}$, and $\mathrm{Lu}^{\mathrm{b}}$ show immature development since their strength and frequency are decreased compared to adults.

The new contribution of the present study may be summarized as follows. 1) Antigens $\mathrm{Rh} 17, \mathrm{Jk}^{3}, \mathrm{Fy}^{3}, \mathrm{Js}^{\mathrm{b}}$, and Emma are fully developed at gestational ages investigated. 2) Antigen $\mathrm{P}$, the high frequency RBC membrane globoside, is absent in $12 \%$ and weakly expressed in $10 \%$ of samples, which are constantly $P_{1}$ negative. 3) Antigen $A_{1}$ as determined by the Dolichos Biflorus lectin is not expressed on fetal $\mathrm{RBC}$ at gestational ages investigated. This finding is in accordance with the documented immaturity of antigen $\mathrm{H}$, the biochemical substrate for $\mathrm{A}$ and $\mathrm{A}_{1}$ antigens. 4) Investigation of the 11 antigens with weak fetal expression on cord blood samples obtained from 72 full-term babies shows intermediate developmental stages between the observed fetal and adult conditions. 5) Since antigens I and $i$ are specific and reliable markers of adult and fetal $\mathrm{RBC}$, provided appropriately adjusted anti-I and anti-i are used, these reagents represent useful and remarkably easy to handle tools to check, by an immediate spin or slide test, for accidental puncture of maternal vessels during fetal blood sampling for prenatal diagnosis procedures. Indeed, other methods are currently available for identification of fetomaternal blood mixtures which are principally based on differential characteristics of fetal and adult hemoglobins. However, these methods are time consuming and do not yield extemporaneous results as those obtained by anti-I and anti-i reagents implemented in this study.

\section{REFERENCES}

1. Race RR, Sanger R 1975 Blood Groups in Man, 6th ed. Blackwell Scientific Publications, London

2. Toivanen P, Hirvonen $T 1973$ Antigens Duffy, Kell, Kidd, Lutheran and $X_{g^{a}}$ on fetal red cells. Vox Sang 24:372-376

3. Mackenzie I, Guest CM, Bowell PJ 1983 Fetal blood group studies during midtrimester pregnancy and the management of severe iso-immunization. Prenat Diagn 3:41-46

4. Daffos F, Capella-Pavlovsky M, Forestier F 1983 Fetal blood sampling via the umbilical cord using a needle guided by ultrasound. Report of 66 cases. Prenat Diagn 3:271-277 